\title{
Can explicit training in Cued Speech improve phoneme identification?
}

Rees, R., Fitzpatrick, C., Foulkes, J., Peterson, H. and Newton, C.

\begin{abstract}
When identifying phonemes in new spoken words, lipreading is an important source of information for many deaf people. Because many groups of phonemes are virtually indistinguishable by sight, deaf people are able to identify about $30 \%$ of phonemes when lipreading nonwords. Cued Speech (CS) is a system of handshapes and hand positions used alongside speech to disambiguate similarities in lippatterns. Deaf children exposed to CS from under 3 years of age go on to develop age-appropriate language and literacy skills. However, there are no studies evaluating the explicit training of CS with older deaf children. This study is the first part of a long-term project to develop and evaluate a computerdelivered programme to teach school-aged deaf children to recognise cued phonemes. Sixty two adult hearing participants were allocated to a single training session in one of three training conditions: Cued Speech Training (CST), Lipreading Training and Auditory Training in Noise. They were all tested on their ability to identify 13 phonemes in nonwords when denied access to sound. The CST group made highly significant improvements in identifying cued phonemes and these changes could not be explained by lipreading practice or familiarisation with a closed set of phonemes. Improvements generalised to nonwords that were not used in training. This suggests that explicit training in CS could help deaf children to identify cued phonemes in new words. Practical implications of introducing the teaching of CS to selected deaf children are discussed.
\end{abstract}

\section{Introduction}

Typically developing hearing children are able to distinguish all phonological contrasts in their spoken language through hearing and vision. This leads to the formation of complete and accurate phonological representations that support spoken language and literacy development (Leybaert, Bayard, Colin, \& LaSasso, 2015). Deaf children rely more heavily on speechreading to distinguish these contrasts and, for many, speechreading is the main source of information (Bernstein, Demorest, \& Tucker, 2000). The term speechreading refers to the extraction of meaning from facial expression, body language and linguistic and situational cues as well as lipreading cues, provided by movements of the lips, jaw and tongue (Arnold, 1997). Bernstein et al. (2000) studied the speechreading skills of deaf and 
hearing adults. When identifying phonemes in Consonant-Vowel nonwords, both groups only succeeded with approximately $30 \%$ phonemes. This is not surprising as there are many groups of phonemes that are virtually indistinguishable by sight (Massaro, 1998). These groups (e.g. $/ \mathrm{p} /, / \mathrm{b} /$ and $/ \mathrm{m} /$ ) are often referred to as visemes as they are deemed to form single perceptual units (Massaro, 1998). Therefore lipreading provides limited information with which to distinguish phonemes. When learning new vocabulary deaf children are relying mainly on lipreading to develop phonological representations, so there is a need to improve children's ability to lipread in order to increase the accuracy of these representations. The study reported here is the first part of a long-term project to help deaf children to lipread new words. It focuses on evaluating a training programme with hearing adults denied access to sound.

Digital hearing aids and cochlear implants can provide deaf children with additional auditory information about speech but the signal is often incomplete. Cochlear implants are still limited in their ability to reproduce the speech perception abilities of the normal ear (Moore, 2003). As a result, even deaf children implanted before 2 years often have difficulties in acquiring aspects of spoken language, especially morphosyntax (Duchesne, 2015). As Duchesne (2015) explains, grammatical morphemes tend to be unstressed and shortened and are visually less salient (Hage, 2005, cited in Duchesne, 2015) and so difficult to identify by lipreading. This is an example of how ambiguous information delivered by lipreading often cannot resolve the limitations of cochlear implants (see Leybaert \& LaSasso, 2010 for a more detailed description of these limitations). This can lead to a reduction in the number and quality of phonological representations that are formed, with subsequent effects on language, phonological awareness and literacy development.

Cued Speech (CS; Cornett, 1967) was designed by Orin Cornett to be an aid to lipreading. $\mathrm{CS}$ is a system of handshapes and hand positions that is used alongside natural speech to distinguish all the phonemes of the spoken language (see Leybaert et al 2015 for a full description). It is designed to be used by those who communicate with deaf children. To distinguish phonemes, the child has to look at the combination of the cues and the lippatterns. For example, consonants that are difficult to discriminate visually (e.g. $/ \mathrm{p} /, / \mathrm{b} /$ and $/ \mathrm{m} /$ ) will have different hand shapes to distinguish them, while consonants with similar hand shapes (e.g. /b/ and /n/) will be easily recognised by lipreading. Unlike systems of hand gestures used to support phonics teaching for literacy, CS does not require the child to have any explicit understanding of the structure of phonological representations. Also, it can be used 
in real time and at the speed of natural speech. Therefore, it is possible for parents and other adults to use the system with a child as soon as they are diagnosed with deafness.

Children exposed to CS at home from under the age of 3 years go on to develop superior phonological awareness skills and literacy skills when compared with matched groups of deaf children not exposed to CS (Bouton, Bertoncini, Serniclaes, \& Colé, 2011; Charlier \& Leybaert, 2000; Crain \& LaSasso, 2010). This early exposure to CS leads to age-appropriate language and literacy skills (Colin, Leybaert, Ecalle, \& Magnan, 2013; Crain \& LaSasso, 2010; Leybaert, 2000; Rees \& Bladel, 2013). Children exposed to CS at a later age at school do not attain these age-equivalent achievements (Charlier \& Leybaert, 2000; Colin et al., 2013) though they do show improvements in speech perception (Nicholls \& Mcgill, 1982). This early study tested the speech perception abilities of 18 deaf children aged from 9 to 16 years, none of whom were exposed to CS before the age of 4 years. One of the assessments required children to identify spoken Consonant-Vowel or Vowel-Consonant syllables in different conditions by writing their orthographic equivalents. Accuracy of identification was compared in three conditions: audition alone, audition with lipreading and audition with lipreading and CS. Accuracy was greater when lipreading was added and, when cues were added, the mean percentage of accuracy rose from 35 to 80 . This indicated that the addition of CS was benefitting the children's ability to distinguish phonemic contrasts. While late introduction to CS may not lead to age-equivalence in terms of the development of phonological representations, significant improvements in speech perception can lead to the updating of existing phonological representations and the formation of more accurate representations for new words being learnt.

School-aged deaf children have the potential to learn CS more explicitly, as they will have better attention than younger children and the ability to reflect on the internal structure of phonological representations. From 6 years of age, children with language impairment seem to benefit from the additional explicit teaching of rules associated with grammatical morphemes (Finestack \& Fey, 2009) and a study by Rees (2009) demonstrated that deaf children aged between 8 and 9 years benefitted from explicit speech training, involving phonological awareness and knowledge of letter-sound correspondences. To date there are no studies that evaluate the explicit teaching of CS perception (recognising cues) to schoolaged deaf children, despite the potential of CS to help older deaf children to learn new words. An efficient way to evaluate the benefit of CS for learning new words is to assess whether learning to perceive cues would increase the ability to identify phonemes in nonwords. Such 
an evaluation could be completed with hearing adults or children denied access to sound, before experimenting with deaf children.

This study is the first part of a project to develop a training programme for the explicit teaching of CS perception to school-aged deaf children. This study evaluates a newly designed training programme delivered via computer with hearing adults denied access to sound, by testing their ability to identify phonemes in cued nonwords without sound before and after the 45-minute training.

Any explicit training in recognising cued phonemes in nonwords presented without sound would involve extra lipreading practice. Hearing adults have been found to gain from explicit lipreading training when recognising phonemes in CV syllables (Gesi, Massaro, \& Cohen, 1992; Massaro, Cohen, \& Gesi, 1993). These training studies involved at least 3 hours of training and so improvements in lipreading may not be expected after a shorter training programme. In evaluating the effects of CS training it is important therefore to control for any gains that would be made in lipreading skills. While improved lipreading abilities could be an additional advantage of CS training it would be important to ensure that gains made were not solely due to these improvements.

As information from cues is combined with lipread information, people with better speechreading skills could potentially benefit more from explicit CS training. It has been established that both the deaf and hearing populations vary widely in their speechreading ability (Auer \& Bernstein, 2007; Bernstein et al., 2000), so the study looked for any association between initial lipreading ability and any improvements in identifying cued phonemes. The term lipreading is used in this context as the tests and training involved single nonwords, so that no cues were provided from facial expressions, body language or linguistic or situational contexts.

The main aim of the study was to investigate whether one 45-minute session of CS training would increase the ability of hearing adults to identify phonemes in cued nonwords presented without sound. Any improvements were correlated with the participants' lipreading scores in the pre-test to look for any association with initial lipreading ability. Any improvements were also compared to changes made in two control programmes. Should the programme prove successful it can be adapted for use with children and trialled with school-aged deaf participants. 


\section{Method}

As the training involved a single session, the phonemes involved in the tests and training were restricted to a closed set of eight consonants and five vowels. In order to assess whether CS training was influencing the identification of cued phonemes it was necessary to compare it to other types of training. To ensure that any improvements were not solely due to lipreading practice, a lipreading programme was included. To avoid any improvements being due to familiarity with a closed set of phonemes, a third auditory training (AT) programme was added. Participants were randomly assigned to one of these three training programmes: Cued Speech Training (CST), LT and AT. All programmes involved the same set of phonemes and the same training techniques (e.g. provision of exemplars and production and perception practice) and lasted a similar length of time: between 40 and 45 minutes. All participants were tested on their ability to identify the phonemes in cued and non-cued nonwords presented without sound before and after training. It was predicted that the CST would lead to a significant increase in the number of cued phonemes correctly identified and the other training programmes would result in no significant changes.

\section{Participants:}

Sixty three participants aged between 21 and 61 years $(\mathrm{M}=28.03$ and $\mathrm{SD}=9.832)$ were recruited through advertisements for volunteers sent to students in a university in the UK. The majority (55) were female. All had English as a first language and 28 spoke additional languages. All reported no difficulties with vision, hearing or literacy. Participants were randomly allocated to the three training groups so that 21 people were trained in each condition. They were given no information about the expectations of the different training programmes before the experiment to avoid influencing the outcomes, but fully briefed at the end of the training.

\section{Test:}

The test involved participants viewing video clips of an adult producing 32 different Consonant-Vowel-Consonant (CVC) nonwords (e.g. /bIm/) without sound. The nonwords were formed from this restricted set of phonemes:

- Consonants: /p/, /b/, /t/, /d/, /m/,/n/, /l/, /f/

- Vowels: /æ/, /e/, /I/, /p/, / / / 
These phonemes were selected for two reasons. They all have highly predictable spellings in CVC combinations. Some consonants were not used in final position (e.g. /1/ and /f/) as they are generally spelt with a double consonant. The majority of consonants were chosen because they are in the same viseme group: the group: $/ \mathrm{p} /, / \mathrm{b} /, / \mathrm{m} /$ and the group $/ \mathrm{t} /, / \mathrm{d} /, / \mathrm{n} /$ and the remaining consonants $(/ 1 /, / \mathrm{f} /)$ were chosen because they are relatively easy to recognise and would ease the challenge of the tasks and may promote confidence.

The 32 nonwords included two matched sets of CVCs that shared the same number of each phoneme but each set had different combinations of these phonemes. One set (of 16 items) was used in the training and the second set was used only in the test. Half of the test items were spoken with cues and half without cues, with the conditions alternating. For example, the first four items were cued and the next four items were not cued. A second version of the test (Version 2) was designed where cued items in Version 1 were not cued and vice versa.

Before viewing the video clips, the participants were told that they would see "nonsense words" spoken with or without hand cues that would be made up of one consonant followed by a vowel and followed by another consonant. They were provided with a list of the written versions of the consonants (p, b, t, d, m, n, l, f) and vowels (a, e, i, o, u) which they could refer to throughout the test. They were told that sounds may be repeated in and across the items.

Participants were asked to write down a CVC combination after viewing each item on the computer screen, and were encouraged to guess if they found it difficult to identify the sounds. Each item was viewed only once and the participants were given 7 seconds in which to provide their responses. The experimenter was present throughout the task.

Participants in each of the three groups were randomly assigned to two different test orders:

1. Version 1 used pre-training and Version 2 used post training

2. Version 2 used pre-training and Version 1 used post training.

For each test, the number of phonemes correctly identified formed a total score out of 96, as there were three phonemes in each nonword. Separate scores were also calculated for the number of phonemes correctly identified for the non-cued items (to assess initial lipreading ability), cued items (to address the main research question), items used in training and items not used in training (to assess generalisation of any learning that took place). 


\section{Training Programmes:}

The three training programmes were: CST, LT and AT. In the CST and LT programmes the video clips of spoken stimuli were presented without sound. In the CST all the stimuli were produced with cues. In the AT programme the video clips were replaced by speech bubbles appearing on the computer screen and the spoken stimuli were presented with white noise added, where the signal to noise ratio was $-10 \mathrm{~dB}$. The addition of noise meant that the hearing participants would have difficulty in identifying the phonemes, but that it was possible to identify some phonemes if they listened intently.

In other ways the three training programmes were designed to be as similar as possible. Each programme played through without a break to ensure that each participant spent the same length of time viewing and interacting with materials. All programmes involved the same set of single phonemes and nonwords with their orthographic equivalents. The 16 treated items were included in all three training conditions. All spoken stimuli were presented the same number of times in each programme. The instructions and explanations in all programmes were presented in the written and spoken form with sound.

All programmes began with an explanation that participants would be trained to perceive the differences between the sounds used in the preceding test and that single sounds and nonsense words would be presented and practised in sets of two or three. The first sets involved single sounds (e.g. /p/ vs. /b/ vs. /m/). See Figure 1 for an example of how single sounds were first presented in the CST programme. A video-clip of one sound being cued was presented with the corresponding written letter appearing below. Then the last frame of this clip and the letter remained on the screen while the next clip and letter appeared. The screenshot in Figure 1 shows the third sound being cued while the final frames and letters of the first two sounds remain on the screen. 


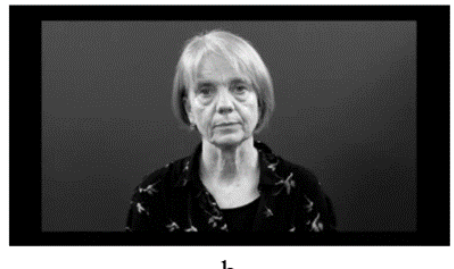

b

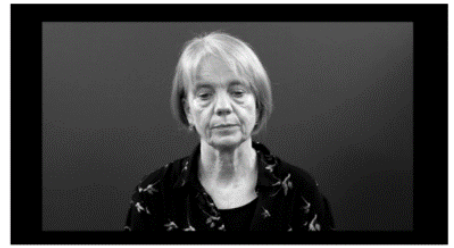

$\mathrm{m}$

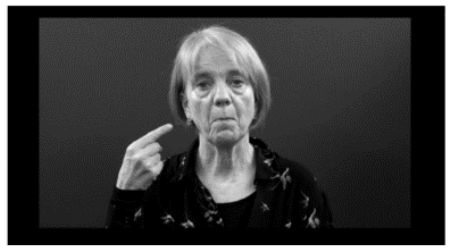

$\mathrm{p}$

Figure 1: Screenshot showing presentation of final sound, while final frames of previous clips and corresponding letters remain on the screen.

The second group of sets involved VC combinations (e.g. /pp/ vs. /ep/). For both these sets there were explanations of the differences between the two or three stimuli in the group. In the CST programme the explanations concerned differences in hand cues and in the LT and AT programmes the explanations concerned differences in articulation.

For example, for /p/ vs. /b/ vs. /m/: the written and spoken explanation in the CST included:

"Note that in the hand cue for " $b$ " no thumb is showing and in the hand cue for " $m$ " the thumb is up"

and the explanation in the LT and AT included:

"When the lip closure for " $p$ " is opened, more air is released than for "b". "m" is a continuous sound."

The last set of stimuli involved groups of three CVC nonwords (e.g. /pæp/ vs. /dem/ vs. /lpn/). No further explanations were provided for these stimuli as all the consonants and vowels used had already been introduced.

For all sets of stimuli presented there were three consecutive stages:

Provision of Exemplars: Each item in a set (e.g. $/ \mathrm{p} /, / \mathrm{b} /$ and $/ \mathrm{m} /$ ) was presented with their written equivalent appearing simultaneously below. This presentation was repeated. This allowed participants to familiarise themselves with how the stimuli were produced with no pressure to identify the items.

Production Practice: Even though the participants were being trained to perceive differences between phonemes, there is evidence to suggest that some practice in producing phonemes can 
improve their identification (Kartushina, Hervais-Adelman, Frauenfelder, \& Golestani, 2015; Rees, 2009). The written forms of each item in the set were presented three times each in a random order. Participants were instructed to produce each item (and cue the item in the case of the CST). The spoken forms (or spoken and cued forms) were presented 3 seconds after each presentation of the written equivalent. This allowed those on the CST to check their guesses and those on the LT and AT to think more about the link between the production and perception of the items.

Perception Practice: The spoken forms of each item in the set were presented three times each in a random order. In the CST and LT the items were video clips of the spoken stimuli without sound and in the AT the items were speech bubbles with the speech presented in noise. Participants were asked to identify each item and then to check their guesses when the written form of the item appeared 3 seconds later.

\section{Post Training Questionnaire:}

When the training and post-test were completed participants were asked to complete a short questionnaire. They were asked to rate the helpfulness of methods used in training on a 5 point scale ranging from unhelpful to very helpful.

The methods they were asked to rate were worded slightly differently according to the programme they had been allocated to. The wording used for the CST programme is provided in this list of the methods that were rated:

- Models of lippatterns and cues,

- Explanations of differences between cues,

- Opportunities to produce the cues,

- Correct lippatterns and cues presented to check attempts at production,

- Opportunities to guess which sounds were being cued,

- Correct letters presented to check guesses.

\section{RESULTS}

First, we established whether the training was effective in improving participants' ability to identify phonemes in cued nonwords. Two one-way ANOVAs were performed in order to 
establish whether the performance of the groups undergoing the three training conditions differed before and after training in response to the cued nonwords. There was no significant difference in performance between groups before training, $\mathrm{F}(2,62)=1.113, \mathrm{p}=.335$, indicating that all groups performed equally accurately prior to receiving training. The results for the post-test showed a main effect of group, $F(2,62)=26.557, p<.001$, indicating that there were differences in accuracy between groups after participants received training. Post hoc tests (Tukey's HSD; $\mathrm{p}<.05$ ) revealed that the scores of the group who received CST were significantly higher than those of both the LT and AT groups. Post-test scores for the LT group were also significantly higher than those of the AT group (see Figure 2).

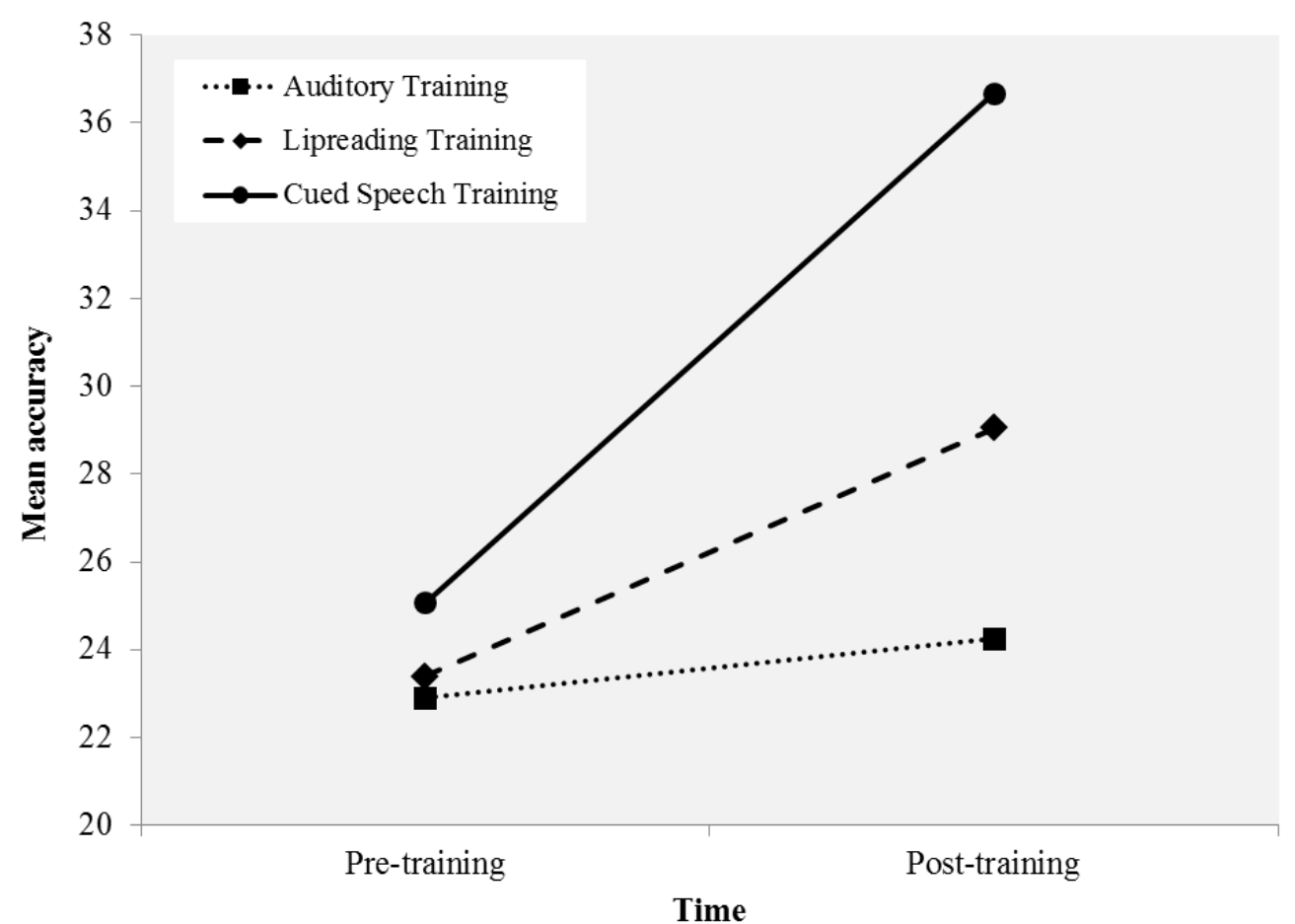

Figure 2. Accuracy in identifying cued phonemes in the pre- and post-tests by training group.

Whereas the AT group showed no significant improvements in their performance on cued nonword items after training, significant improvements were found in both the CST group $(t(20)=-9.812, p<.001)$ and the LT group $(t(20)=-5.342, p<.001)$.

To establish whether the increase in identification accuracy in the group who had received CST could be generalised to cued nonwords not included in the training programme, we conducted an additional paired comparison on just the untreated cued nonwords for the CST 
group, comparing before $(\mathrm{M}=12.667, \mathrm{SD}=2.331)$ and after training $(\mathrm{M}=17.667, \mathrm{SD}=$ 3.838 ), finding a significant increase in identification accuracy, $t(20)=6.642, p<.001$.

We also investigated whether the improvements made by the CST group were as a result of the training in identifying cues or were due to increased attention to lip movements in training. If the latter were true, we would also expect a significant improvement after training for the non-cued nonwords (those spoken and not cued) included in the pre-/post-test. A related samples t-test revealed an improvement only approaching significance, $t(20)=-1.794$, $p=.088$ (see Figure 3). Comparison of pre- and post-test scores for cued nonwords was highly significant, $t(20)=-9.812, p<.001$. Further analysis confirmed that improvements made for cued nonwords (i.e. post-test score minus pre-test score) were significantly greater than those for non-cued nonwords, $t(40)=-7.248, p<.001$.

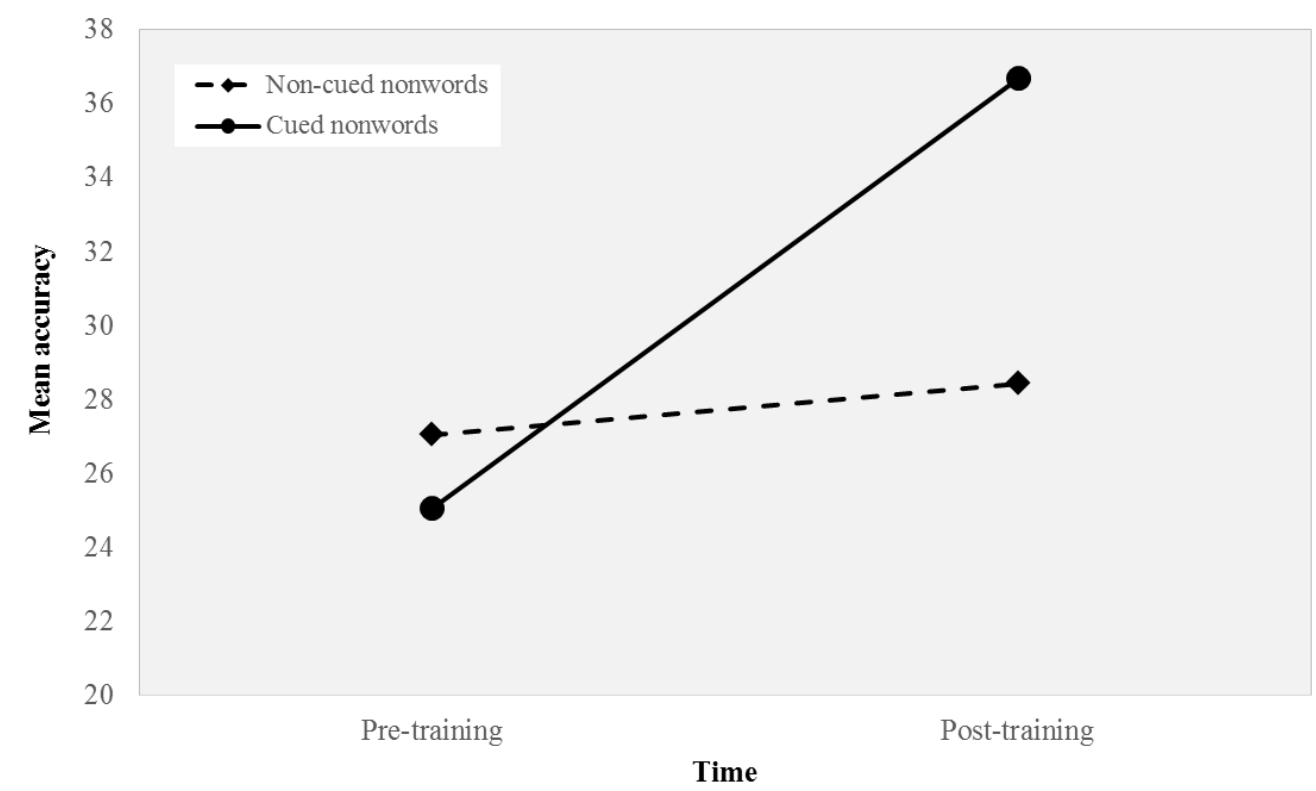

Figure 3. Accuracy in identifying phonemes in non-cued and cued nonwords before and after CST

Finally, in order to establish whether improvement in identifying cued phonemes after training was related to participants' existing lipreading ability, we compared their scores on the non-cued items in the pre-training test with improvements made in identifying phonemes in cued nonwords (as measured by the difference in accuracy before and after training). This correlation was not significant, $r=.269, p=.238$. 


\section{Post-Training Questionnaire:}

The average ratings for each training method given by the 21 participants who completed the CST programme are shown in Table 1.

$\begin{array}{ll}\text { TRAINING METHOD } & \text { AVERAGE RATING } \\ & (1=\text { Unhelpful, 5=Very Helpful })\end{array}$

\begin{tabular}{ll}
\hline Models of lippatterns and cues & 4.50 \\
\hline Explanations of differences between cues & 3.71 \\
\hline Opportunities to produce the lippatterns and cues & 4.00 \\
\hline Correct lippatterns and cues presented to check & 4.57 \\
attempts at production & 4.67 \\
\hline Opportunities to guess which sounds were being cued & 4.67 \\
\hline Correct letters presented to check guesses & \\
\hline
\end{tabular}

Table 1. CST programme: Average ratings for training method

\section{Discussion}

This study showed that one single session of Cued Speech Training (CST) significantly improved participants' ability to identify cued phonemes in nonwords presented without sound. This concurs with the finding that CS enhances the speech perception of nonwords for older deaf children with very limited use of hearing (Nicholls \& Mcgill, 1982). In this early study, the children's average score when identifying nonwords with cues was $80 \%$, with a range from 59 to $96 \%$. In this study the participants' average score for identifying cued phonemes post-training was $37 \%$, with a range from 27 to $48 \%$. However, although the deaf children in the early study had not been introduced to CS before the age of 4 years, they had been exposed to CS implicitly from between 5 and 10 years and were also making use of amplification of their residual hearing. The hearing adult participants in this study had only 45 minutes to learn the cues for 13 phonemes and had no auditory cues. In this short time they averaged an $11 \%$ improvement in their ability to identify cued phonemes, with a range from 3 to $21 \%$. Therefore it is likely that, with a longer training programme, the improvement could continue.

Findings also suggested that the significant improvement made by participants in the CST group applied to both the cued CVC items used in training and those not used in training, 
demonstrating generalisation. This transfer of learning to other combinations of phonemes does suggest that the participants were learning to discriminate and identify phonemes rather than nonwords as whole units.

The improvements made in the CST could not be accounted for by familiarisation with the restricted set of 13 phonemes. The AT programme required the participants to consider the same set of phonemes in very similar exercises for the same length of time and this group made no significant improvement in their ability to identify cued phonemes.

An unexpected finding was that the LT lead to significant improvements in the participants' ability to identify cued phonemes. It is unlikely that this was due to practice from the pretraining test as it lasted less than 5 minutes and only half the items were cued and those were not cued in the post-training test. Previous studies evaluating lipreading training for nonwords with hearing adults involved at least 3 hours of training (Gesi et al., 1992; Massaro et al., 1993). However, in a similar study with deaf adults, it was noted that, although the training programme lasted up to 14 hours, most of the improvements were made in the first few hours (Walden, Prosek, Montgomery, Scherr, \& Jones, 1977). The deaf participants in this study had learnt to distinguish some of the phonemes, where there were visual cues to use. Walden et al. (1977) gave the example of participants who were unable to distinguish $/ \mathrm{t} /, / \mathrm{d} /, \mathrm{s} /$ and $\mathrm{z} /$ before training learning to distinguish /t, d/ vs. /s, z/ after some training and who were likely to be using duration cues. Therefore it is possible that participants in the LT group quite quickly learned to distinguish some groups of phonemes, where visual information was available. However, as stated by Gesi et al. (1992), some nonword syllables may be visually indistinguishable, which can lead to limited improvements in lipreading training for most people. That is likely to explain the superior performance of the CST group in identifying cued phonemes in the post-training test, as CS cues are designed specifically to disambiguate lipreading similarities (Cornett, 1967).

Participants in the CST group also had better scores for identifying non-cued phonemes after the training, although the difference was only approaching significance. These participants were given no information about how the sounds were articulated; this was only provided to those in the LT group. This slight but non-significant improvement concurs with the results of the training study conducted by Gesi et al (1992). Hearing adult participants were randomly allocated to two different types of lipreading training lasting 3 hours: both involved forced choice practice with feedback but only one provided information about where to look 
and what to look for when trying to distinguish phonemes. Both groups learnt with training and there were no differences between the effects of the two types of training. Therefore practice in lipreading with no explanation could lead to some modest improvements in lipreading.

Even though the participants receiving CST made some modest, non-significant gains in identifying non-cued phonemes, their improvement in identifying cued phonemes was highly significant. Additionally, improvements for cued nonwords were significantly greater than improvements for non-cued nonwords. This suggests that the addition of cues helped participants to identify phonemes after one session of training and that this improvement could not solely be explained by improvements in lipreading. This concurs with findings that deaf children exposed to CS from an early age can become skilled at lipreading non-cued speech (Rees \& Bladel, 2013) and a likely explanation is the necessity to combine information from cues and lippatterns; children learning to perceive CS have to pay attention to lippatterns as well as cues. A case study of a nine-year-old deaf boy exposed to CS from the age of one year showed that he had learnt to lipread non-cued familiar language competently, making very few errors. However, he still benefitted significantly from the addition of cues when identifying phonemes in nonwords (Rees \& Bladel, 2013).

Findings from the current study suggest that it is possible to train those with no or limited hearing to benefit from the addition of CS when identifying phonemes in nonwords. Nonwords are comparable with new words that are being learnt, as the learner has no stored lexical representation of the word to aid recognition. The combination of phonemes in nonwords is unfamiliar and, due to the ambiguities of lipreading, it is often difficult for a deaf child to identify each of the phonemes. Therefore, the late learning of CS could help deaf children to identify phonemes in new words and store them accurately in their lexicon.

CS could also help deaf children to update inaccurate phonological representations of words already stored. Deaf children often develop inaccurate phonological representations due to limited auditory information and, although information from orthography can help the older children to update these representations, it can also lead to non-standard forms (Leybaert, 1993). Leybaert (1993) gives the example of deaf children naming a drawing of "tabac" (tobacco) as [tabak] even though it is an irregular French word that is pronounced as [taba]. Rees (2009) gives the example of a nine-year-old deaf child pronouncing the English word 
"stomach" as [d $\wedge \mathrm{m} \Lambda \mathrm{P}]]$. This pronunciation error could be explained by a phonological representation that was formed due to limited auditory and lipreading information and misleading orthographic information.

School-aged deaf children with inaccurate phonological representations and difficulties in identifying the phonemes in new words could potentially benefit from explicit training in CS. To benefit from the kind of training used in this study they would ideally have to be familiar with letter-sound correspondences and be able to segment words into phonemes.

Lipreading ability is known to vary widely in both the hearing and deaf populations (Auer \& Bernstein, 2007; Bernstein et al., 2000) and so it is possible that older deaf children with better lipreading skills would benefit more from training in CS. However, in this study, no association was found between initial lipreading ability for non-cued items and improvements made in identifying cued phonemes. This suggests that training in CS could benefit deaf children with a range of lipreading abilities.

The CST used in this study was effective but, in order to trial with deaf children, it would need to be adapted, with the addition of more interactive exercises, engaging materials and motivating rewards. Feedback from participants receiving CST indicated that most methods used were helpful. However, a limitation of the questionnaire was that there was a bias in the rating scale in that three out of five ratings were positive. This may have affected the responses although the relative degree of usefulness would not be affected. The majority of participants found the following methods mostly helpful or very helpful: models of lippatterns and cues, opportunities to produce the cues, correct lippatterns and cues presented to check attempts at production, opportunities to guess which sounds were being cued and correct letters presented to check guesses. Even though participants knew they were being trained to perceive cues, they still found it helpful to practise producing cues. This concurs with findings from other studies indicating that some practice in producing spoken phonemes can improve their identification (Kartushina, Hervais-Adelman, Frauenfelder, \& Golestani, 2015; Rees, 2009). Participants found perception practice marginally more helpful. The programme used in this study had an equal balance of production and perception practice. Future adaptations could alter the balance and provide more perception practice. Explanations of differences between cues were reported to be the least helpful but still reported to be sometimes helpful or mostly helpful by the majority. Comparisons of lipreading training methods that include and exclude explanations of differences have found 
no significant differences in results. This does suggest that participants may learn to distinguish differences without needing explanations.

The next stage of this project will be to adapt the current CST programme for use with children and to trial the updated programme with hearing school-aged children denied access to sound. If this second stage has positive results, the same programme will be trialled with school-aged deaf children.

This study has shown that one 45-minute session of explicit training to perceive CS resulted in a significant improvement in the identification of cued phonemes for hearing adults who were denied access to sound. This improvement was found for untrained nonwords as well as trained nonwords. The highly significant improvements could not be explained by familiarity with a closed set of phonemes or practice in lipreading. Therefore, an adapted CST programme could help school-aged deaf children to identify cued phonemes, allowing them to update any inaccurate phonological representations and form more accurate representations for new words being learnt.

\section{Acknowledgements}

The authors would like to thank all the participants and give special thanks to Dr Mike Coleman, UCL, London, who developed software for the computer programmes.

\section{References}

Arnold, P. (1997). The Structure and Optimization of Speechreading. Journal of Deaf Studies and Deaf Education, 2(4), 199-211.

Auer, E. T., \& Bernstein, L. E. (2007). Enhanced Visual Speech Perception in Individuals With Early-Onset Hearing Impairment. Journal of Speech, Language, and Hearing Research, 50(5), 1157-1165. https://doi.org/10.1044/1092-4388(2007/080)

Bernstein, L. E., Demorest, M. E., \& Tucker, P. E. (2000). Speech perception without hearing. Perception and Psychophysics, 62(2), 233-252.

Bouton, S., Bertoncini, J., Serniclaes, W., \& Colé, P. (2011). Reading and Reading-Related Skills in Children Using Cochlear Implants: Prospects for the Influence of Cued 
Speech. Journal of Deaf Studies and Deaf Education, 16(4), 458-473. https://doi.org/10.1093/deafed/enr014

Charlier, B. L., \& Leybaert, J. (2000). The rhyming skills of deaf children educated with phonetically augmented speechreading. The Quarterly Journal of Experimental Psychology A, 53(2), 349-375. https://doi.org/10.1080/027249800390529

Colin, S., Leybaert, J., Ecalle, J., \& Magnan, A. (2013). The development of word recognition, sentence comprehension, word spelling, and vocabulary in children with deafness: a longitudinal study. Research in Developmental Disabilities, 34(5), 17811793.

Cornett, O. (1967). Cued Speech. American Annals of the Deaf, 112, 2-13.

Crain, K. L., \& LaSasso, C. . (2010). Generative rhyming ability of 10- to 14-year-old readers who are deaf from oral and cued speech backgrounds. In C. J. LaSasso, K. L. Crain, \& J. Leybaert (Eds.), Cued speech and cued language for deaf and hard of hearing children. San Diego: Plural Publishing, 345-358.

Duchesne, L. (2015). Grammatical Competence After Early Cochlear Implantation. In M. Marschark \& P. E. Spencer (Eds.), The Oxford Handbook of Deaf Studies in Language. Oxford University Press, 113-132.

Finestack, L. H., \& Fey, M. E. (2009). Evaluation of a Deductive Procedure to Teach Grammatical Inflections to Children With Language Impairment. American Journal of Speech-Language Pathology, 18(3), 289-302. https://doi.org/10.1044/10580360(2009/08-0041)

Gesi, A. T., Massaro, D. W., \& Cohen, M. M. (1992). Discovery and Expository Methods in Teaching Visual Consonant and Word Identification. Journal of Speech Language and Hearing Research, 35(5), 1180-1188. https://doi.org/10.1044/jshr.3505.1180 
Kartushina, N., Hervais-Adelman, A., Frauenfelder, U. H., \& Golestani, N. (2015). The effect of phonetic production training with visual feedback on the perception and production of foreign speech sounds. The Journal of the Acoustical Society of America, 138(2), 817-832. https://doi.org/10.1121/1.4926561

Leybaert, J. (1993). Reading Ability and Phonological Codes. In M. Marschark \& M. D. Clark (Eds.), Psychological Perspectives on Deafness. Hillsdale, New Jersey: Lawrence Erblaum Associates, 269-309.

Leybaert, J. (2000). Phonology Acquired through the Eyes and Spelling in Deaf Children. Journal of Experimental Child Psychology, 75(4), 291-318. https://doi.org/10.1006/jecp.1999.2539

Leybaert, J., Bayard, C., Colin, C., \& LaSasso, C. (2015). Cued Speech and Cochlear Implants: A Powerful Combination to Natural Spoken Language Acquisition and the Development of Reading. In M. Marschark \& P. E. Spencer (Eds.), The Oxford Handbook of Deaf Studies in Language. Oxford University Press, 359-376.

Leybaert, J., \& LaSasso, C. J. (2010). Cued Speech for Enhancing Speech Perception and First Language Development of Children With Cochlear Implants. Trends in Amplification, 14(2), 96-112. https://doi.org/10.1177/1084713810375567

Massaro, D. (1998). Perceiving Talking Faces: From Speech Perception to a Behavioural Principle. Cambridge: MIT Press.

Massaro, D. W., Cohen, M. M., \& Gesi, A. T. (1993). Long-term training, transfer, and retention in learning to lipread. Perception \& Psychophysics, 53(5), 549-562. https://doi.org/10.3758/BF03205203

Moore, B. C. J. (2003). Coding of Sounds in the Auditory System and Its Relevance to Signal Processing and Coding in Cochlear Implants. [Miscellaneous Article]. Otology \& Neurotology, 24(2), 243-254. 
Nicholls, G. H., \& McGill, D. (1982). Cued Speech and the reception of spoken language. Journal of Speech Language and Hearing Research, 25(2), 262-269. https://doi.org/10.1044/jshr.2502.262

Rees, R. (2009). Deaf children's acquisition of speech skills: a psycholinguistic perspective through intervention. Doctoral Thesis, UCL. Available from: http://discovery.ucl.ac.uk/1348395.

Rees, R., \& Bladel, J. (2013). Effects of English Cued Speech on Speech Perception, Phonological Awareness and Literacy: A Case Study of a 9-Year-Old Deaf Boy Using a Cochlear Implant. Deafness \& Education International, 15(4), 182-200. https://doi.org/10.1179/1557069X13Y.0000000025

Walden, B. E., Prosek, R. A., Montgomery, A. A., Scherr, C. K., \& Jones, C. J. (1977). Effects of Training on the Visual Recognition of Consonants. Journal of Speech Language and Hearing Research, 20(1), 130-145.

https://doi.org/10.1044/jshr.2001.130 